\title{
Characteristics of infarction after encephaloduroarteriosynangiosis in young patients with moyamoya disease
}

\author{
Hyun Gi Kim, MD, PhD, ${ }^{1,2}$ Seung-Koo Lee, MD, PhD, ${ }^{1}$ and Jung-Dong Lee, MS ${ }^{3}$ \\ 1Department of Radiology and Research Institute of Radiological Science, Yonsei University College of Medicine, Seoul; and \\ 2Department of Radiology and ${ }^{3}$ Office of Biostatistics, Ajou University School of Medicine, Suwon, Korea
}

\begin{abstract}
OBJECTIVE Young patients with moyamoya disease can exhibit infarction after revascularization surgery. This analysis of the characteristics of infarction after encephaloduroarteriosynangiosis (EDAS) in young patients with moyamoya disease was undertaken in an effort to elucidate the infarction mechanism.

METHODS The authors retrospectively collected clinical information and reviewed pre- and postoperative MRI studies from cases involving patients younger than 18 years who underwent EDAS for the treatment of moyamoya disease between January 2012 and February 2015. Infarction patterns were categorized into watershed, territorial, or mixed pattern. The Wilcoxon rank sum test, chi-square test, and Fisher exact test were used to compare the clinical and imaging variables between patient groups. The characteristics of patients with and without postoperative infarction were compared using univariate and multivariate analysis. The cumulative proportion of patients without postoperative infarction according to operation stage was calculated using the Kaplan-Meier method and the resulting curves were compared using the log-rank test.
\end{abstract}

RESULTS In 100 patients, 171 EDAS procedures had been performed. There were 38 cases of preoperative infarction in 35 patients and 20 cases of postoperative infarction in 13 patients. Territorial infarction was more frequent in the postoperative infarction group than in the preoperative infarction group $(55.0 \%$ vs $37.8 \%, p=0.037)$. Infarction was more common on the bilateral or contralateral side of the operation after first-stage EDAS (9 [75.0\%] of 12 infarctions) than in the second-stage operation (2 [25.0\%] of 8 infarctions), but the difference was not statistically significant $(p=0.068)$. The frequency of postoperative infarction was not significantly different depending on the stage of the operation $(p=0.694)$.

CONCLUSIONS An acute infarction pattern after EDAS was more frequently territorial, suggesting an underlying occlusive mechanism. Operation stage did not affect the rate of postoperative infarction occurrence.

http://thejns.org/doi/abs/10.3171/2016.7.PEDS16218

KEY WORDS moyamoya disease; revascularization; cerebral infarction; complication; pediatric; infarction pattern; operation stage; vascular disorders

$\mathrm{M}$ OYAMOYA disease is the most common cerebrovascular disease in Eastern Asian children. The disease is characterized by progressive stenosis or occlusion of the internal carotid artery or its branches. Depending on the stage of disease, extensive collateral vessels develop around the circle of Willis. Several different surgical revascularization procedures may be offered to patients with symptomatic moyamoya disease, including direct bypass surgery from the superficial temporal artery to distal branches of the anterior cerebral artery (ACA) or middle cerebral artery (MCA) or indirect bypass surgery such as encephaloduroarteriosynangiosis (EDAS) or encephalodurogaleo (periosteal) synangiosis., ${ }^{4,18,23}$ Among the procedures, indirect bypass surgeries are performed more often in children with ischemic symptoms, as the procedures are easier to perform and have high rates of successful revascularization. ${ }^{6,16}$ After surgical procedures, proper collateral circulation can be achieved in most patients. $2,10,21$

Even though surgical intervention is the treatment of

ABBREVIATIONS $\mathrm{ACA}$ = anterior cerebral artery; $\mathrm{EDAS}=$ encephaloduroarteriosynangiosis; $\mathrm{MCA}=$ middle cerebral artery; $\mathrm{PCA}=$ posterior cerebral artery; $\mathrm{TIA}=$ transient ischemic attack; TOF = time of flight.

SUBMITTED April 19, 2016. ACCEPTED July 14, 2016

INCLUDE WHEN CITING Published online October 7, 2016; DOI: 10.3171/2016.7.PEDS16218. 
choice for symptomatic young moyamoya patients, the possibility of postoperative ischemia exists. Studies have been performed to identify risk factors for postoperative infarction, and age younger than 3 years and preoperative infarction have been suggested as major risk factors. ${ }^{5,13}$ Attempts to identify a postoperative infarction mechanism have led to the proposal of a progressive occlusion of the main cerebral arteries ${ }^{14}$ or "watershed shift" phenomenon. ${ }^{7}$ By evaluating angiographic pattern and infarction location on angiography, MR angiography, or positron emission tomography-CT, progressive occlusion of the main cerebral arteries ${ }^{14}$ and interference with antegrade flow due to newly developed anastomoses ${ }^{7}$ were proposed as possible infarction mechanisms after surgery.

Another way to investigate postoperative infarction mechanisms might be by looking into the pattern of infarction. One study of patterns of cerebral infarction in children with moyamoya disease found that preoperative infarction was characterized by a watershed pattern, suggesting a nonvasoocclusive mechanism. ${ }^{24}$ Although there are reports of the preoperative infarction pattern, ${ }^{1,24}$ we know of no reports on the pattern of infarction after EDAS that will elucidate the mechanism of postoperative infarctions. In addition, no study has shown whether there is any difference in postoperative infarction characteristics according to operation stage. Therefore, the purpose of our study was to clarify the characteristics of postoperative infarction in young patients with moyamoya disease, focusing on the most widely used indirect anastomosis procedure, EDAS.

\section{Methods}

The institutional review board of Severance Hospital approved this retrospective study and waived the need for informed consent.

\section{Patients}

Between January 2012 and February 2015, 118 pediatric patients underwent EDAS in our institution. This group included 18 patients who had moyamoya syndrome (moyamoya phenomena caused by underlying disease) and were excluded from the study. The remaining 100 patients had idiopathic moyamoya disease and were included in the study. Of these 100 children and adolescents, 26 underwent unilateral EDAS. Among the 74 patients who underwent second-stage EDAS, there were 3 patients who underwent only second-stage EDAS during the study period. Therefore, 171 EDAS procedures in 100 children with idiopathic moyamoya disease were included for study population.

\section{Operative Technique and Postoperative Treatment}

A surgical team performed all of the operations using a modified EDAS procedure as previously described. ${ }^{13}$ The outer layer of the dura mater, including the middle meningeal artery, was attached to the cortical surface of the brain. Surgery was performed in 2 stages: 1) in the hemodynamically affected and symptomatic hemisphere; and 2) in the contralateral hemisphere. The 2 staged procedures were performed at least 2 weeks apart. The intra- operative $\mathrm{PaCO}_{2}$ was maintained at $35-45 \mathrm{~mm} \mathrm{Hg}$. The mean arterial blood pressure was maintained at $100-120$ $\mathrm{mm} \mathrm{Hg}$. Continuous delivery of fentanyl $(0.5-1.5 \mathrm{mg} / \mathrm{kg} /$ hour) was provided to control postoperative pain.

\section{Clinical Variables}

The patients' medical records were reviewed to collect information related to the surgical procedures and the known clinical risk factors for postoperative infarction. Patient age $(<3$ years or $\geq 3$ years $),{ }^{5,13}$ sex,${ }^{12}$ preoperative neurological deficit (absent or present), preoperative symptom side (absent, unilateral, or bilateral), and the interval between the onset of transient ischemic attacks (TIAs) and the operation were collected. Operative information including the stage (first or second) and side of the operation (right or left) was collected. For patients with postoperative infarction, the number of days between the operation and infarction were calculated. The frequency of TIAs and pre- or postoperative seizures, which are known clinical risk factors for postoperative infarction, ${ }^{13}$ were excluded from the variable set since the data were missing in a large number of patients.

\section{Imaging Variables}

All patients who receive surgical treatment for moyamoya disease in our institution undergo preoperative MRI within 7 days before surgery. Preoperative MRI includes T2-weighted, diffusion-weighted, and time-of-flight (TOF) imaging. Based on the results of imaging, preoperative staging was assessed using Suzuki angiographic criteria. ${ }^{26}$ Both Suzuki stage and the presence of posterior cerebral artery (PCA) stenosis were evaluated using both TOF and 3D reconstructed MR angiography images. Preoperative collaterals were assessed with preoperative MRI. Since only 8 of the 100 patients in the study group underwent conventional catheter angiography before surgery, collateral assessment on conventional angiography was excluded from the imaging variables. The presence of preoperative infarction, infarction side, and infarction pattern (watershed, territorial, or mixed) were evaluated.

After surgery, diffusion-weighted MR images were obtained in cases in which there was a clinical suspicion of postoperative infarction. Postoperative infarction, a primary interest variable, was defined as newly developed diffusion restriction on MR images obtained after the operation. We included cases of acute infarction within 30 days after surgery. ${ }^{25}$ Postoperative infarction pattern (watershed, territorial, or mixed), side, shape (punctate, gyral, or other), and concordance between operation and infarction side (ipsilateral or bi-/contralateral) were evaluated. A territorial infarction pattern was further characterized as an ACA, MCA, or PCA territorial infarction.

\section{Operation Stage and Postoperative Infarction}

To investigate whether there was a difference in postoperative infarction characteristics according to the operation stage, the number of days between the operation and infarction, the side and pattern of infarction, and the concordance between operation side and infarction (ipsilateral or bi-/contralateral) were compared between first- and 
second-stage postoperative infarction cases. Infarctions only on the ipsilateral side of the operation were categorized as ipsilateral side infarctions. Infarctions in the bilateral hemisphere or contralateral side were categorized as bilateral or contralateral side infarctions. The rates of postoperative infarction after the first- and second-stage operations were compared.

\section{Statistics}

The Wilcoxon rank sum test, chi-square test, and Fisher exact test were used to compare the clinical and imaging variables between patient groups according to the presence of postoperative infarction, pre- or postoperative infarction, and operation stage. The Wilcoxon rank sum test was used to compare continuous variables, including the interval between TIA onset and surgical treatment and the interval between the operation and infarction. Chi-square and Fisher exact tests were used to compare other categorical variables. We performed a Fisher exact test for Suzuki stage and PCA stenosis to identify the extent of association between these 2 variables. As Suzuki stage and PCA stenosis were highly associated ( $\mathrm{p}<0.001)$, we excluded Suzuki stage as an independent variable. All the other variables that showed a significant difference between the 2 groups (i.e., cases with and without postoperative infarction) were included in the variables for univariate and multivariate analysis. The odds ratios for postoperative infarction for clinical and imaging findings were calculated (with 95\% confidence intervals) with univariate and multivariate logistic regression analyses. As most patients underwent a 2-stage operation, we used logistic regression with a generalized estimating equation (GEE). When preoperative and postoperative infarction patient groups were compared, we included patients who had both preoperative and postoperative infarction. The Kruskal-Wallis test was used for analyzing differences in the number of days between the operation and postoperative infarction among the 3 infarction-pattern groups. The cumulative proportion of patients without postoperative infarction according to operation stage was calculated by Kaplan-Meier's method and the differences were assessed for statistical significance with the log-rank test. A p value less than 0.05 was considered statistically significant. SAS version 9.4 (SAS Inc.) and R package version 3.1.2 (R Foundation for Statistical Computing) were used for statistical analysis.

\section{Results}

Forty-three boys and 57 girls were included in this study. Their mean age was 7.9 years (range 1-17 years). There were 20 postoperative infarctions in 13 patients, but 2 patients suffered 2 episodes of infarction within the first 30 days after a single EDAS procedure. Thus the infarctions occurred after 18 procedures. The frequency of infarction was $13 \%$ calculated on a per patient basis (13 of 100 patients) and $10.5 \%$ calculated on a per procedure basis (18 of 171 of EDAS procedures). Of the 13 patients who suffered postoperative infarctions, 6 had mild neurological deficits and 7 had moderate to severe neurological deficits after surgery.

When the group of operations followed by infarction (n $=18$ ) and the group that were not associated with postoperative infarction $(n=153)$ were compared, the following variables were significantly associated with postoperative infarction: patient age less than 3 years $(33.3 \%$ vs $5.2 \%$, p $<0.001$ ), shorter interval between TIA onset and operation ( 2 months vs 6 months, $\mathrm{p}=0.028$ ), higher Suzuki stage (Stage IV, 38.8\% vs $11.8 \%, \mathrm{p}=0.016$ ), presence of PCA stenosis ( $44.4 \%$ vs $12.4 \%, \mathrm{p}<0.001)$, and presence of preoperative infarction $(55.6 \%$ vs $17.6 \%, \mathrm{p}<0.001)$. Among 28 cases with PCA stenosis, 11 had preoperative infarction. On univariate analysis to evaluate the association between clinical and imaging findings and postoperative infarction, age less than 3 years was the factor with the most significant association (Table 1). On multivariate analysis, age less than 3 years (OR 38.08, 95\% CI 4.72-307.29, p < 0.001 ) and PCA stenosis on preoperative MRI (OR 12.13, 95\% CI 2.03-72.53, $\mathrm{p}=0.006$ ) had significant associations with postoperative infarction.

Clinical and imaging characteristics in preoperative and postoperative infarctions are shown in Table 2 . There were 38 preoperative infarctions in 35 patients and 20 postoperative infarctions in 13 patients (Fig. 1). In terms of infarction pattern, territorial infarction was the dominant pattern in the postoperative infarction group (11 [55.0\%] of 20 infarctions) and watershed pattern was the dominant pattern in the preoperative infarction group (21 [56.8\%] of 38 infarctions). The frequency of territorial infarction was significantly higher in the postoperative infarction group $(55.0 \%$ vs $37.8 \%, \mathrm{p}=0.037)$. Among 11 postoperative territorial infarctions, 7 were MCA territory infarctions, and

TABLE 1. Results of univariate and multivariate analyses of association between clinical and imaging findings and postoperative infarction

\begin{tabular}{|c|c|c|c|c|}
\hline \multirow[b]{2}{*}{ Variable } & \multicolumn{2}{|c|}{ Univariate Analysis } & \multicolumn{2}{|c|}{ Multivariate Analysis } \\
\hline & $\mathrm{OR}(95 \% \mathrm{Cl})$ & $p$ Value & $\mathrm{OR}(95 \% \mathrm{Cl})$ & $\mathrm{p}$ Value \\
\hline \multicolumn{5}{|l|}{ Clinical findings } \\
\hline Age <3 yrs & $9.69(2.20-42.62)$ & 0.003 & $38.08(4.72-307.29)$ & $<0.001$ \\
\hline Male sex & $0.82(0.24-2.78)$ & 0.752 & $0.35(0.05-2.20)$ & 0.260 \\
\hline Interval btwn TIA onset \& op & $0.99(0.93-1.05)$ & 0.684 & $1.00(0.96-1.04)$ & 0.928 \\
\hline 2nd op & $0.76(0.35-1.63)$ & 0.479 & $0.64(0.18-2.31)$ & 0.496 \\
\hline \multicolumn{5}{|l|}{ Imaging findings } \\
\hline Preop infarction & $2.60(0.92-7.32)$ & 0.071 & $1.54(0.28-8.44)$ & 0.619 \\
\hline PCA stenosis & $3.82(0.42-34.80)$ & 0.235 & $12.13(2.03-72.53)$ & 0.006 \\
\hline
\end{tabular}


TABLE 2. Comparison of clinical and imaging findings in preoperative and postoperative infarction

\begin{tabular}{|c|c|c|c|}
\hline Variable & $\begin{array}{c}\text { Preop } \\
\text { Infarction }\end{array}$ & $\begin{array}{l}\text { Postop } \\
\text { Infarction }\end{array}$ & $\mathrm{p}$ Value \\
\hline Age & & & 0.078 \\
\hline$<3$ yrs & $4(10.5 \%)$ & $6(10.5 \%)$ & \\
\hline$\geq 3$ yrs & $34(89.5 \%)$ & $14(89.5 \%)$ & \\
\hline Sex & & & 0.407 \\
\hline Male & $21(55.3 \%)$ & $8(40.0 \%)$ & \\
\hline Female & $17(44.7 \%)$ & $12(60.0 \%)$ & \\
\hline Preop neurologic deficit & & & 0.999 \\
\hline No & $37(97.4 \%)$ & $20(100.0 \%)$ & \\
\hline Yes & $1(2.6 \%)$ & $0(0.0 \%)$ & \\
\hline Side of preop symptoms & & & 0.933 \\
\hline No & $1(2.8 \%)$ & $1(5.0 \%)$ & \\
\hline Right & $13(36.1 \%)$ & $6(30.0 \%)$ & \\
\hline Left & $17(47.2 \%)$ & $11(47.2 \%)$ & \\
\hline Both & $5(13.9 \%)$ & $2(13.9 \%)$ & \\
\hline \multicolumn{4}{|c|}{ Interval btwn TIA onset \& op } \\
\hline Median & $5 \mathrm{mos}$ & $1 \mathrm{mo}$ & 0.120 \\
\hline IQR & $1-24$ mos & $1-6 \mathrm{mos}$ & \\
\hline Suzuki stage & & & 0.127 \\
\hline I or II & $0(0.0 \%)$ & $1(5.0 \%)$ & \\
\hline III & $29(76.3 \%)$ & $11(55.0 \%)$ & \\
\hline IV & $9(23.7 \%)$ & $8(40.0 \%)$ & \\
\hline PCA stenosis & & & 0.351 \\
\hline No & $27(71.1 \%)$ & $11(55.0 \%)$ & \\
\hline Yes & $11(29.0 \%)$ & $9(45.0 \%)$ & \\
\hline Infarction side & & & 0.999 \\
\hline Right & $19(51.4 \%)$ & $10(50.0 \%)$ & \\
\hline Left & $9(24.3 \%)$ & $5(25.0 \%)$ & \\
\hline Both & $9(24.3 \%)$ & $5(25.0 \%)$ & \\
\hline Pattern of infarction & & & 0.037 \\
\hline Watershed & $21(56.8 \%)$ & $5(25.0 \%)$ & \\
\hline Territorial & $14(37.8 \%)$ & $11(55.0 \%)$ & \\
\hline Mixed & $2(5.4 \%)$ & $4(20.0 \%)$ & \\
\hline Side of op & & & 0.999 \\
\hline Right & $20(52.6 \%)$ & $10(50.0 \%)$ & \\
\hline Left & $18(47.4 \%)$ & $10(50.0 \%)$ & \\
\hline
\end{tabular}

$\mathrm{IQR}=$ interquartile range .

1 was an ACA territory infarction. Two postoperative territorial infarctions included an ACA territory infarction in one hemisphere and an MCA territory infarction in the other hemisphere. In 1 case, the infarction was localized at the basal ganglia. There was no PCA territory infarction. The mean duration between surgery and postoperative infarction was 16 days (range 1-28 days), 9 days (range 0-30 days), and 3 days (range 1-6 days) in the watershed, territorial, and mixed pattern infarction groups, respectively. There was no statistically significant difference in the number of days between surgery and postoperative infarction for the 3 infarction pattern groups $(\mathrm{p}=0.180)$.

Clinical and imaging findings of postoperative infarc- tions after first- and second-stage operations are compared in Table 3. For infarctions occurring after first-stage operations, the infarction was more frequently bilateral or contralateral to the operation side (9 [75.0\%] of 12 infarctions) (Fig. 1A and B) compared with the second-stage operation (2 [25.0\%] of 8 infarctions) (Fig. 1C and D), but the difference was not statistically significant $(p=0.068)$. There was no significant difference in the number of days between the operation and postoperative infarction, pattern of infarction, or shape of infarction according to operation stage. The infarction shape was mostly gyral for infarctions occurring after either stage. Five patients among 8 patients who had infarction after the second-stage operation had already had infarction after the first-stage operation. The cumulative proportion of patients without postoperative infarction did not differ significantly depending on the operation stage $(\mathrm{p}=0.694)$ (Fig. 2).

\section{Discussion}

\section{Frequency of and Risk Factors for Postoperative Infarction}

Unlike adult patients, pediatric patients can benefit from indirect revascularization procedures, and among such procedures, EDAS is widely accepted as the preferred surgery for pediatric moyamoya patients. ${ }^{6}$ The frequency of infarction after EDAS in our study (10.5\%) is consistent with the previous reports for indirect surgery $(2.9 \%-11.9 \%) .{ }^{11}$ In our study, independent factors associated with infarction after EDAS were age less than 3 years and presence of PCA stenosis on preoperative MRI. This is consistent with previous reports suggesting risk factors of postoperative infarction of age under 3 years, ${ }^{5,13}$ PCA involvement, ${ }^{9}$ female sex ${ }^{12}$ frequent TIAs,${ }^{13}$ underlying disease, ${ }^{5,15}$ preoperative infarction on imaging studies, ${ }^{13}$ recent stroke, ${ }^{13}$ and higher angiographic stage. ${ }^{9}$ Kim et al. showed a higher frequency of preoperative infarction in the group with postoperative infarction $(9 / 12,75.0 \%$, vs $82 / 158,51.9 \%, \mathrm{p}<0.01) .{ }^{13}$ In that study, there were 4 cases of PCA stenosis (33.3\%) among 12 postoperative infarction cases and 67 cases of PCA stenosis (42.4\%) among 158 cases without postoperative infarction. The frequency of PCA stenosis in the 2 groups was not statistically different. In our study, however, the presence of PCA stenosis was an independent risk factor for postoperative infarction and the presence of preoperative infarction was not.

\section{Characteristics of Postoperative Infarction and Proposed Mechanisms}

The ischemic patterns in young patients with moyamoya disease are distinct from those in adult patients with moyamoya disease, suggesting different hemodynamic characteristics and vulnerability. ${ }^{1}$ Analysis of preoperative infarction patterns has pointed to nonvasoocclusive ischemic mechanisms in pediatric moyamoya patients. ${ }^{24}$ However, there is a lack of understanding of postoperative infarction mechanisms in these patients. Since the postoperative infarction pattern may suggest its underlying pathophysiology, which may be different from the preoperative infarction pattern in young moyamoya patients, we analyzed postoperative infarction characteristics after 

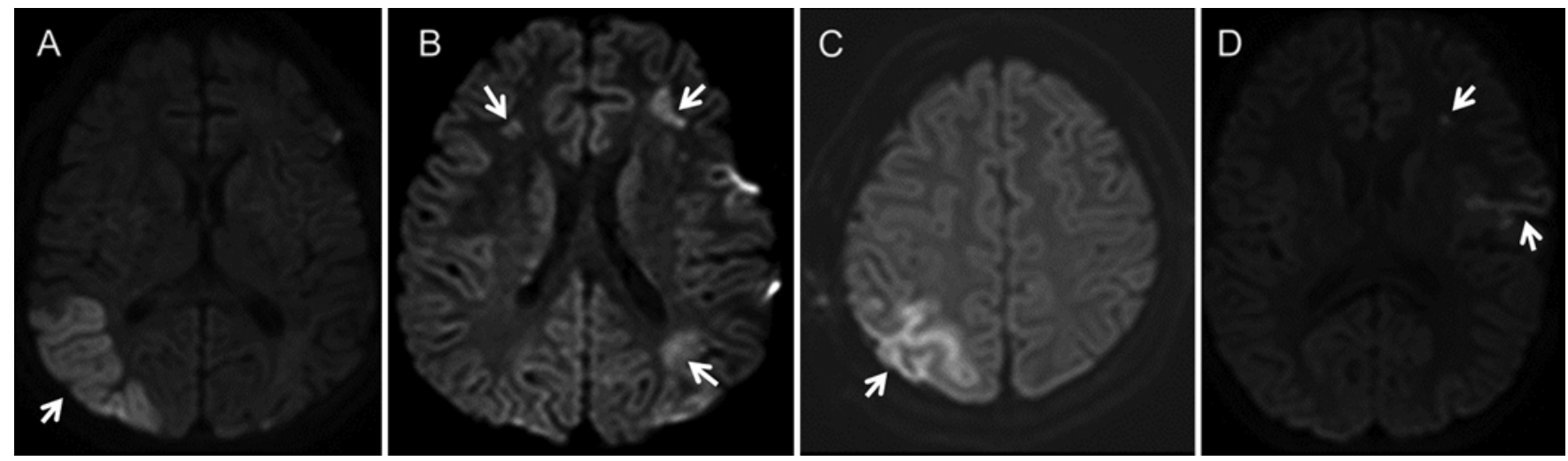

FIG. 1. Representative axial diffusion-weighted images showing examples of postoperative infarction (arrows) of different patterns and affected sides. A: Image obtained in a 2-year-old girl after first-stage EDAS on the left hemisphere showing right MCA territorial infarction. B: Image obtained in a 9-year-old boy after first-stage EDAS on the left hemisphere showing bilateral watershed infarction. C: Image obtained in a 13-year-old girl after second-stage EDAS on the right hemisphere showing right MCA territorial infarction D: Image obtained in a 5-year-old boy after second-stage EDAS on the left hemisphere showing left MCA territorial and watershed infarction.

EDAS and compared them with those of preoperative infarctions.

Our results showed that territorial infarction was the major infarction pattern in postoperative infarctions. Moreover, the territory of infarction was MCA in most cases, which is different from the ACA territorial infarction shown in a previous study by Kuroda et al. suggesting a mechanism of postoperative infarction. ${ }^{14}$ Kuroda et al. proposed that progressive occlusion of the main cerebral arteries and decreased anterior flow from the decreased moyamoya vessels are the main reasons for postoperative infarction in moyamoya disease patients with frontal lobe infarction..$^{14}$ However, only 2 adult patients who underwent both direct and indirect surgical procedures were included in the report. Therefore, disagreement may be attributable to differences in the patient population and surgery type. Another proposed mechanism of postoperative infarction in patients with moyamoya disease is the "watershed shift" phenomenon proposed by Hayashi et al., ${ }^{7}$ which can explain MCA territory infraction. Based on findings of SPECT and MRI, Hayashi et al. postulated that antegrade flow from the proximal MCA that supplies distal and deeper areas of the MCA territory could be interfered with by the bypass from the direct anastomosis. Thus, this mechanism may explain the ipsilateral territorial infarction in our study. Besides, indirect surgery alone could interfere with antegrade MCA flow to the temporal surface since edema from the craniotomy site could induce edema of the brain. ${ }^{3}$

Yet, as our results showed postoperative infarctions on the side contralateral to the operation side, a local effect from surgery cannot alone explain the postoperative infarction mechanism. Instead, more systemic change in hemodynamics should be considered as the cause of infarction. It is well known that the maintenance of normocapnia with normotension is important for the perioperative management of patients with moyamoya disease. ${ }^{8}$ The anesthetic management in these patients has evolved over the years to reduce perioperative complications, ${ }^{22}$ and patients in our study also received careful anesthetic management to reduce perioperative infarction during and after surgery. Still, there are doubts as to whether the hemodynamics of all patients could be controlled after the surgery as strictly as during the surgery. Certain hemodynamic changes, therefore, may provoke progressive occlusion of the artery, which is a generally plausible mechanism of infarction in moyamoya disease.

\section{Operation Stage and Postoperative Infarction}

To the best of our knowledge, no study has shown an effect of operation stage on infarction characteristics. Our results showing a greater frequency of bilateral or contralateral infarctions after first-stage EDAS might be because the predominantly symptomatic side (with respect to TIAs) was selected for the first operation. It is common in pediatric moyamoya disease for a single patient to have

TABLE 3. Comparison of clinical and imaging findings in cases of infarction occurring after first- and second-stage operations

\begin{tabular}{llll}
\hline \multicolumn{1}{c}{ Variable } & 1st Stage & 2nd Stage & p Value \\
\hline No. of postop infarctions & 12 & 8 & \\
\hline Interval btwn op \& infarction (days) & $5(1-12)$ & $9(2-17)$ & 0.876 \\
\hline Side of postop infarction & & & 0.075 \\
\hline Right & $4(33.0 \%)$ & $6(75.0 \%)$ & \\
\hline Left & $3(25.0 \%)$ & $2(25.0 \%)$ & \\
\hline Both & $5(41.7 \%)$ & $0(0.0 \%)$ & \\
\hline Pattern of postop infarction & & & 0.586 \\
\hline Watershed & $2(16.7 \%)$ & $3(37.5 \%)$ & \\
\hline Territorial & $7(58.3 \%)$ & $4(50.0 \%)$ & \\
\hline Mixed & $3(25.0 \%)$ & $1(12.5 \%)$ & \\
\hline Shape of infarction & & & 0.308 \\
\hline Punctate & $2(16.7 \%)$ & $0(0.0 \%)$ & \\
\hline Gyral & $6(50.0 \%)$ & $7(87.5 \%)$ & \\
\hline Others & $4(33.3 \%)$ & $1(12.5 \%)$ & \\
\hline Op \& infarction side & & & 0.068 \\
\hline Ipsilateral & $3(25.0 \%)$ & $6(75.0 \%)$ & \\
\hline Bilateral or contralateral & $9(75.0 \%)$ & $2(25.0 \%)$ & \\
\hline
\end{tabular}




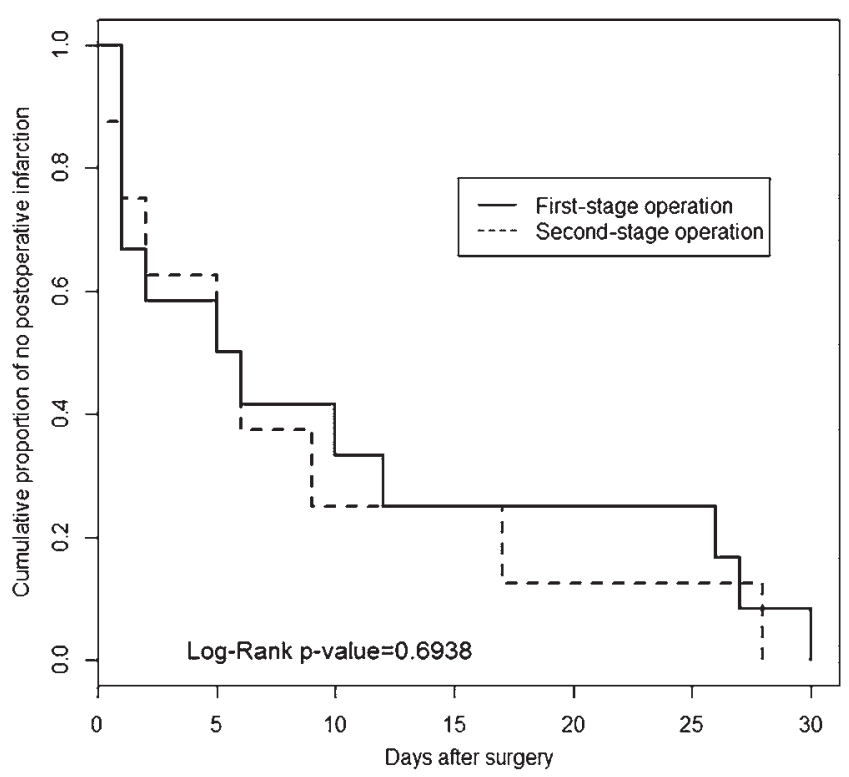

FIG. 2. Kaplan-Meier plot of the effect of operation stage on postoperative infarction.

different stages of disease in the left and right sides. ${ }^{17,19}$ Operation on the more hemodynamically unstable side may more induce a loss of moyamoya vessels, which may eventually affect cerebral blood flow in the opposite hemisphere. Conversely, since the hemisphere treated in the first operation becomes more hemodynamically stable after that operation, and its moyamoya vessels have already been reduced, it is less likely to be affected by the second operation, when it is the contralateral hemisphere.

Accordingly, we assumed that the postoperative infarction rate would be higher after first-stage operations because the first-stage operation is on the predominantly symptomatic side. However, we did not find any statistically significant difference between the first- and secondstage operations with respect to postoperative infarction rates. This might be because the patients who had significant infarctions after a first-stage operation may not have undergone a second-stage operation. Although most of the patients (74\%) underwent both first- and second-stage operations, $26 \%$ of the patients underwent only first-stage EDAS during the study period. There are still disagreements concerning the prophylactic value of bypass surgery on the asymptomatic side as well. ${ }^{20}$ We wanted to evaluate whether patients with a postoperative infarction after the first-stage operation will have additional infarction risk after the second-stage operation, but the number of cases of infarction after a second-stage operation $(n=8)$ was too small for the analysis. Still, given that $62.5 \%$ (5/8) of patients who had infarctions after a second-stage operation were those who already had postoperative infarction after the first-stage operation, more caution might be needed for patients who had infarction after the first-stage operation and are undergoing a subsequent second-stage operation.

\section{Limitation}

This study was retrospective, and we could have missed some of the postoperative infarction cases involving pa- tients without significant or detectable symptoms who did not undergo MRI subsequent to infarction. In addition, we could not include all the known risk factors for postoperative infarction, since some variables were missing from the medical charts at the time of surgery. Since our hospital is a tertiary hospital and many EDAS procedures are performed each year, general postoperative management is strictly controlled with respect to temperature, blood pressure, pain control, and $\mathrm{PaCO}_{2}$, but we cannot follow all the various parameters that may differ among patients. Including the missed variables and detailed postoperative management parameters in the analysis could have shown different aspects of postoperative infarction. Nevertheless, this study includes a large number of patients who underwent the same surgical procedure in a single institution. A prospective study analyzing all the known clinical and imaging variables should be performed in the future.

\section{Conclusions}

The infarction pattern after EDAS was more frequently territorial, indicating that postoperative infarction is probably due to an occlusive mechanism rather than hypoperfusion. We hoped to find different trends of infarction according to operation stage, but there was no significant difference between postoperative infarction characteristics after first- and second-stage EDAS. In addition, the stage of operation did not affect the occurrence of postoperative infarction. Knowing the characteristics of postoperative infarction may help improve the results of surgical treatment for patients with moyamoya disease.

\section{References}

1. Cho HJ, Jung YH, Kim YD, Nam HS, Kim DS, Heo JH: The different infarct patterns between adulthood-onset and childhood-onset moyamoya disease. J Neurol Neurosurg Psychiatry 82:38-40, 2011

2. Choi JU, Kim DS, Kim EY, Lee KC: Natural history of moyamoya disease: comparison of activity of daily living in surgery and non surgery groups. Clin Neurol Neurosurg 99 (Suppl 2):S11-S18, 1997

3. Fujimura M, Kaneta T, Shimizu H, Tominaga T: Cerebral ischemia owing to compression of the brain by swollen temporal muscle used for encephalo-myo-synangiosis in moyamoya disease. Neurosurg Rev 32:245-249, 2009

4. Fujita K, Tamaki N, Matsumoto S: Surgical treatment of moyamoya disease in children: which is more effective procedure, EDAS or EMS? Childs Nerv Syst 2:134-138, 1986

5. Funaki T, Takahashi JC, Takagi Y, Kikuchi T, Yoshida K, Mitsuhara T, et al: Unstable moyamoya disease: clinical features and impact on perioperative ischemic complications. J Neurosurg 122:400-407, 2015

6. Fung LWE, Thompson D, Ganesan V: Revascularisation surgery for paediatric moyamoya: a review of the literature. Childs Nerv Syst 21:358-364, 2005

7. Hayashi T, Shirane R, Fujimura M, Tominaga T: Postoperative neurological deterioration in pediatric moyamoya disease: watershed shift and hyperperfusion. J Neurosurg Pediatr 6:73-81, 2010

8. Iwama T, Hashimoto N, Yonekawa Y: The relevance of hemodynamic factors to perioperative ischemic complications in childhood moyamoya disease. Neurosurgery 38:11201126, 1996

9. Jung YJ, Ahn JS, Kwon DH, Kwun BD: Ischemic complications occurring in the contralateral hemisphere after surgical 
treatment of adults with moyamoya disease. J Korean Neurosurg Soc 50:492-496, 2011

10. Kashiwagi S, Kato S, Yamashita K, Takasago T, Akimura T, Okamura S, et al: Revascularization with split duro-encephalo-synangiosis in the pediatric moyamoya disease-surgical result and clinical outcome. Clin Neurol Neurosurg 99 (Suppl 2):S115-S117, 1997

11. Kazumata K, Ito M, Tokairin K, Ito Y, Houkin K, Nakayama $\mathrm{N}$, et al: The frequency of postoperative stroke in moyamoya disease following combined revascularization: a single-university series and systematic review. J Neurosurg 121:432440, 2014

12. Khan N, Achrol AS, Guzman R, Burns TC, Dodd R, BellStephens T, et al: Sex differences in clinical presentation and treatment outcomes in Moyamoya disease. Neurosurgery 71:587-593, 2012

13. Kim SH, Choi JU, Yang KH, Kim TG, Kim DS: Risk factors for postoperative ischemic complications in patients with moyamoya disease. J Neurosurg 103 (5 Suppl):433-438, 2005

14. Kuroda S, Houkin K, Nunomura M, Abe H: Frontal lobe infarction due to hemodynamic change after surgical revascularization in moyamoya disease - two case reports. Neurol Med Chir (Tokyo) 40:315-320, 2000

15. Mallory GW, Bower RS, Nwojo ME, Taussky P, Wetjen NM, Varzoni TC, et al: Surgical outcomes and predictors of stroke in a North American white and African American moyamoya population. Neurosurgery 73:984-991, 981-982, 2013

16. Manceau E, Giroud M, Dumas R: Moyamoya disease in children. A review of the clinical and radiological features and current treatment. Childs Nerv Syst 13:595-600, 1997

17. Matsushima T, Inoue T, Natori Y, Fujii K, Fukui M, Hasuo $\mathrm{K}$, et al: Children with unilateral occlusion or stenosis of the ICA associated with surrounding moyamoya vessels"unilateral" moyamoya disease. Acta Neurochir (Wien) 131:196-202, 1994

18. Matsushima T, Inoue T, Suzuki SO, Fujii K, Fukui M, Hasuo $\mathrm{K}$ : Surgical treatment of moyamoya disease in pediatric patients-comparison between the results of indirect and direct revascularization procedures. Neurosurgery 31:401-405, 1992

19. Mugikura S, Takahashi S, Higano S, Shirane R, Sakurai Y, Yamada S: Predominant involvement of ipsilateral anterior and posterior circulations in moyamoya disease. Stroke 33:1497-1500, 2002

20. Nagata S, Matsushima T, Morioka T, Matsukado K, Mihara F, Sasaki T, et al: Unilaterally symptomatic moyamoya dis- ease in children: long-term follow-up of 20 patients. Neurosurgery 59:830-837, 2006

21. Nakashima H, Meguro T, Kawada S, Hirotsune N, Ohmoto $\mathrm{T}$ : Long-term results of surgically treated moyamoya disease. Clin Neurol Neurosurg 99 (Suppl 2):S156-S161, 1997

22. Parray T, Martin TW, Siddiqui S: Moyamoya disease: a review of the disease and anesthetic management. J Neurosurg Anesthesiol 23:100-109, 2011

23. Patel NN, Mangano FT, Klimo P Jr: Indirect revascularization techniques for treating moyamoya disease. Neurosurg Clin N Am 21:553-563, 2010

24. Rafay MF, Armstrong D, Dirks P, MacGregor DL, deVeber G: Patterns of cerebral ischemia in children with moyamoya. Pediatr Neurol 52:65-72, 2015

25. Scott RM, Smith JL, Robertson RL, Madsen JR, Soriano SG, Rockoff MA: Long-term outcome in children with moyamoya syndrome after cranial revascularization by pial synangiosis. J Neurosurg 100 (2 Suppl Pediatrics):142-149, 2004

26. Suzuki J, Takaku A: Cerebrovascular "moyamoya" disease. Disease showing abnormal net-like vessels in base of brain. Arch Neurol 20:288-299, 1969

\section{Disclosures}

The authors report no conflict of interest concerning the materials or methods used in this study or the findings specified in this paper.

\section{Author Contributions}

Conception and design: Kim. Acquisition of data: Kim. Analysis and interpretation of data: Kim. Drafting the article: Kim. Reviewed submitted version of manuscript: all authors. Approved the final version of the manuscript on behalf of all authors: SK Lee. Statistical analysis: JD Lee. Study supervision: SK Lee.

\section{Supplemental Information}

Previous Presentations

This study was presented at RSNA 2015 (the 101st annual meeting of the Radiological Society of North America), November 29-December 4, 2015.

\section{Correspondence}

Seung-Koo Lee, Department of Radiology and Research Institute of Radiological Science, Yonsei University College of Medicine, 50-1 Yonsei-ro, Seodaemun-gu, Seoul 120-752, Korea. email: slee@yuhs.ac. 